\title{
An Accelerometer Based Sensor Platform for Insitu Elite Athlete Performance Analysis
}

\author{
Daniel A. James \\ Centre for Wireless Monitoring and \\ Applications, \\ CRC for Microtechnology \\ Griffith University, Australia, \\ d.james@griffith.edu.au
}

\author{
Neil Davey \\ Centre for Wireless Monitoring \\ and Applications, \\ CRC for Microtechnology \\ Griffith University, Australia, \\ n.davey@griffith.edu.au
}

\author{
Tony Rice \\ Dept of Physiology, \\ CRC for Microtechnology \\ Australian Institute of Sport \\ Australia \\ tony.rice@ausport.gov.au
}

\begin{abstract}
A sensor data acquisition platform has been developed for in-situ sporting applications encompassing stand alone high speed sampling and storage of multiple accelerometer data. Ambulatory monitoring of elite athlete in competition or training environments was then undertaken. The platform itself is divided into functional blocks and controlled by a small microcontroller using a custom OS. Hence it is easily customizable for a variety of sporting applications. The platform is packaged so as to be robust, hermetically sealed and biomechanically neutral to the athlete.

Results and derivative data from rowing and swimming are presented as sample applications. In swimming applications, stroke characteristics for a variety of training strokes are analyzed and found to be better than hand timed data and comparable with results obtained from technology and labor intensive methods, including video analysis. The rowing application when coupled with other monitoring techniques such as impeller velocity, enables recovery of intra and inter stroke phases as a means to assess performance over the entire course and has been used by competition rowers to improve performance at national and international competitions.
\end{abstract}

\section{Keywords}

Sensor systems, sports, accelerometer. microprocessor

\section{INTRODUCTION}

The performance of elite athletes today arguably has almost as much to do with science, as it does with training. Today athletes, coaches and sport scientists strive to extract incremental 'fraction of a second' improvement in timed events, this often being the difference between today's champion and a finisher. Traditionally the measurement of elite athlete performance is commonly done in a laboratory environment [1], where rigorous testing of physiology can take place. Laboratory testing however necessarily places limits on how the athlete performs, as the environment is sufficiently different to the training environment. Further performance characteristics are further augmented during competition when compared to regular training. By better understanding athlete performance during the competition environment coaches can more effectively work with athletes to improve their performance. Key factors affecting an athlete that are difficult to replicate in the laboratory include the physical environment as well as the mental one. For example it is well understood that runner gait is quite different for treadmill running when compared to track gait characteristics.

The testing and monitoring of elite athletes in their natural training environment is a relatively new area of development that has been facilitated by advancements in microelectronics and other micro technologies. Whilst it is a logical progression to take laboratory equipment and miniturize it for the training and competition environment, it introduces a number of considerations that need to be addressed.

In testing athletes outside a highly controlled laboratory environment we need to consider a number of factors and trade offs, these include what are we going to test or measure, what technologies we can use to obtain the measure, the practicality of obtaining the measure, and others related to the specific sport under consideration. In the case of swimming for example some of the considerations are the weight of the apparatus, if it is too heavy it will be a hindrance to the swimmer. Size and shape also have an effect on hydrodynamics of the device and thus the swimmer. Also what metrics are likely to be practically obtainable by a submersible device in an aquatic environment.

The aspects that need considering when looking to obtain athlete information outside the laboratory environment are heavily influenced by, and reliant on, what technology is currently available. Through technological innovation and development, new sensors, devices and systems become available, coupled with this development is a general trend for reductions in size, power requirements, and cost. The reductions in size and power are very important factors when considering sport specific systems, as elite athletes can be very sensitive in relation to the perception of size and weight of wearable systems.

\section{SENSOR PLATFORM}

The use and application of accelerometers to measure activity levels for sporting[2], health and for gait analysis [3] is emerging as a popular method of biomechanical quantification of health and sporting activity. With the availability of MEMS based accelerometers like the ADXL202 from Ana- 
log Devices and widely available low power microprocessors, a modular sensor platform previously developed by the authors was customized for swimming and rowing applications [4].

Utilization of a sensor platform greatly simplifies the process of measuring a physical event and turning it into something useful (Figure 1) by providing, support for the sensor, basic signal conditioning, storage and application specific processing of the data. In the case of sporting applications this modular approach allows rapid customization and modification as the technical expertise, understanding and expectations of sport scientists was found to develop rapidly along with prototype systems developed for testing. Accelerometers when combined in such a system have enabled the recording of athlete activity and the storage and/or transmission of data.

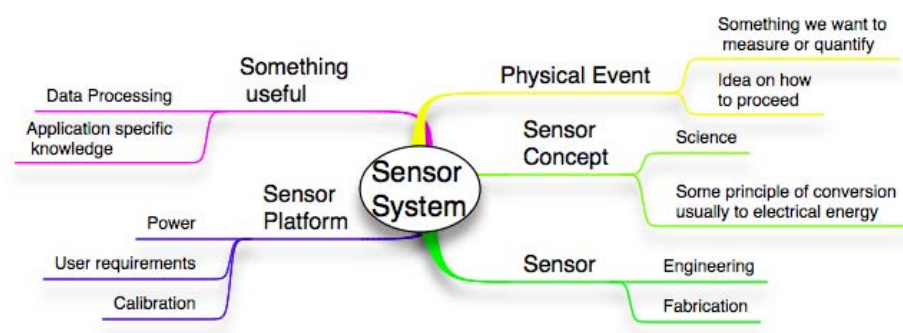

Figure 1 : The role of the sensor platform in sensing applications

The platform supported the acquisition and processing of tri-axial accelerometers at or above $250 \mathrm{~Hz}$ per channel. Data acquisition, basic processing and storage was handled by a custom OS [5] on a $16 \mathrm{MHz}$ microprocessor. Depending on the exact application input from other sensors was also incorporated.

In each case the customized sensor platform was packaged according to the environmental demands of the sport and where appropriate near real time transmission or display of the data was available for coach and athlete feedback.

\section{RESULTS}

Two sports are presented here in as sample applications of the platform, though a number of others are also under development. Rowing was the first sport investigated, as it is (somewhat simplistically) a 1-D sporting activity, following that swimming was selected as the next logical progression in terms of complexity. Currently running and coactive sports are also under investigation.

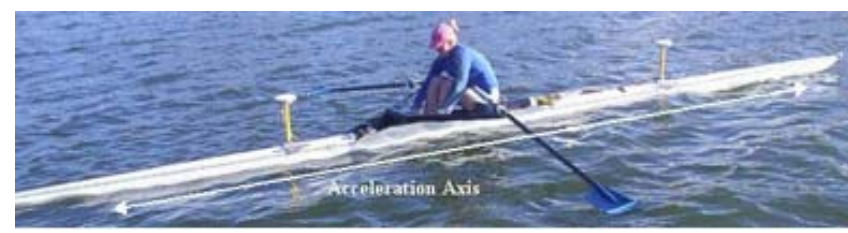

Figure 2: A single rowing scull showing the principle axis of interest

\section{Rowing}

The use of impellers to record scull velocity is somewhat common place, the developed sensor platform incorporated this measurement together with (initially) 1-D accelerometer measurement (see Figure 2), heart beat (using a polar type wireless chest sensor). More recently 3D accelerometers have been used to aid more precise stroke phase determination and GPS has also been employed for more precise position determination. Output from the platform to a palm top computer is facilitated using a serial port (Figure 3 ) to facilitate immediate visual feedback.

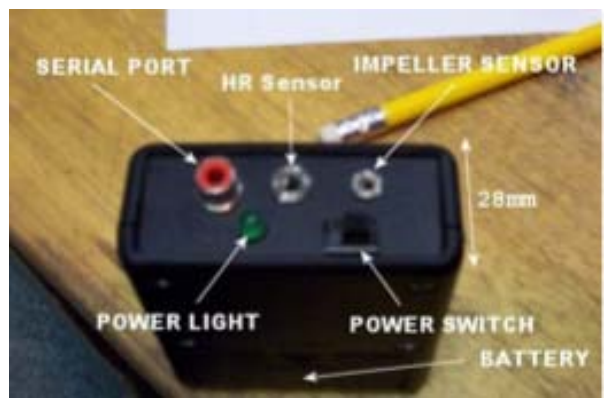

Figure 3: Rowing Platform showing I/O

Key metrics like stroke rate can be derived from real time data and displayed on the palm top computer for immediate feedback to the athlete and it can be viewed using a telemetry module for immediate (and post event) use by coaches and sports scientists. Post activity analysis and stroke diagnosis has been useful for Australian teams to compare inter team differences thus helping to refine and develop race strategies. Figures 4 below shows basic accelerometer traces with the release and catch phases clearly identified. Statistical information derived from these phases of motion are useful benchmarks for coaches that have traditionally been difficult to obtain without purpose instrumented boats and/or video analysis.

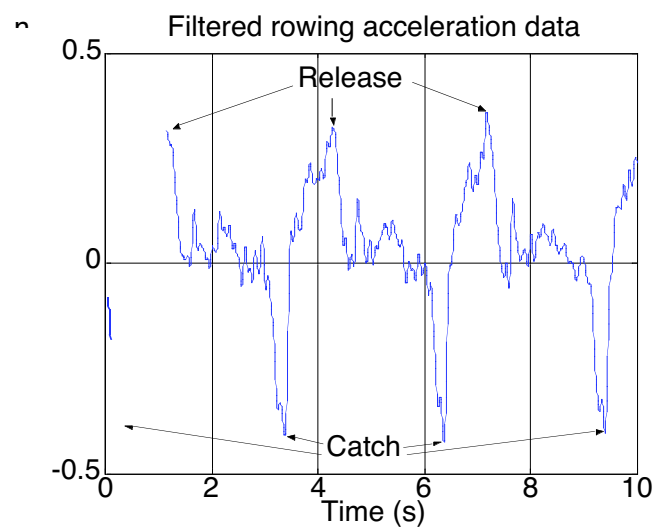

Figure 4: Acceleration activity data recorded during rowing activity 


\section{Swimming}

Although swimming has the principle component of activity in a single direction information contained in the other axis of movement are critical in identifying stroke characteristics and thus performance. For sports analysis swimming is traditionally a labour intensive process where stroke rates, counts and times are often manually recorded and thus only dependant on high staffing levels. A sensor platform for swimming applications also presents significant packaging challenges, as it is required to operate in the aquatic environment and had to be comfortable to wear. The developed design included infra-red communications for data download, intuitive single button operation and inductive charging, thus removing the necessity of physical electrical contact to the device needing to be made. After several iterations the entire platform was encapsulated in a rubberized material and shaped so as to be more comfortable for the athlete (see Figure 5).

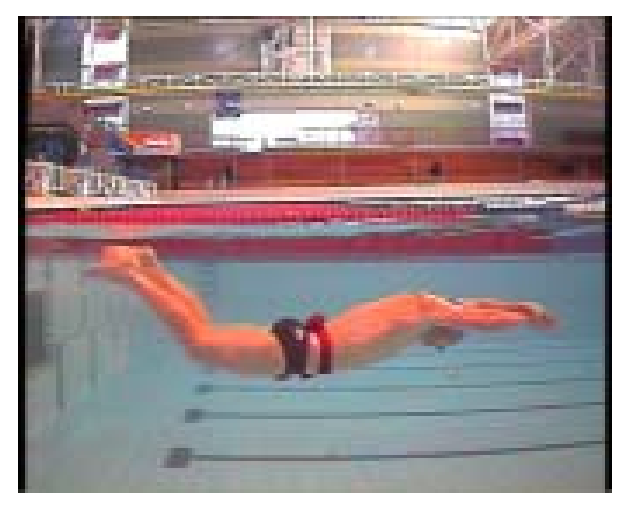

Figure 5: Location of swimming platform for insitu use.

Acceleration data collected from the platform was used to develop data processing algorithms to extract wall push off, stroke type and stroke count metrics. Figure 6 shows some typical raw data collected for the freestyle stroke for a single lap of activity.

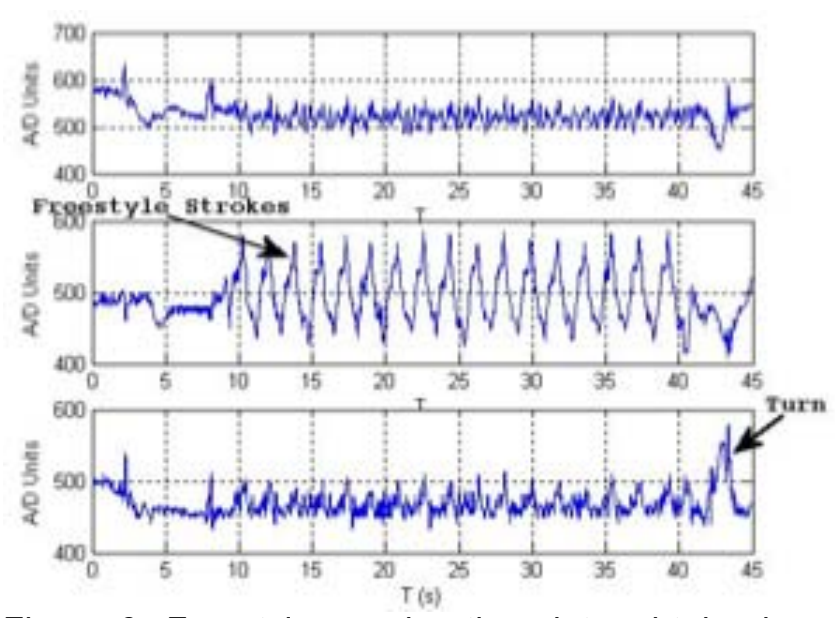

Figure 6: Freestyle acceleration data obtained using the platform

Initially the algorithms were developed using hand timed data and underwater video as benchmarks. At national training camps acceleration data was collected from a representative selection of swimmers (male and female) with the platform together with traditionally obtained metrics. The results of the wall push off algorithm were compared against manual hand timing with $90 \%$ algorithm results being with \pm 1 second of the hand timing data. The stroke type identification algorithm determines which stroke is being swum presently has an accuracy of $95 \%$. The results of the freestyle stroke count algorithm were compared against manual stroke counts from raw accelerometers data and underwater video. Of the 164 data sets analyzed over $90 \%$ of the algorithm results were within \pm 1 strokes of the manual recorded stroke counts.

\section{DISCUSSION AND CONCLUSIONS}

A sensor platform has been customized for use in two elite athlete sports, rowing and swimming. These platforms have been extensively trialed with Australian athletes in their preparations for the 2004 Athens Olympics. Processing techniques were been developed that provided data usually only available to sport scientists, coachs and athletes at intensive training camps. Whilst traditionally data collection has been labour and technology (underwater cameras) intensive, these devices offer the potential to be distributed to all athletes for use in all training sessions.

Further development of processing algorithms is underway in these and other sporting applications. It is clear anecdotally that contained within recorded accelerometer data are signatures and indicators for fatigue, efficacy of training drills and quantitative assessment of what ideal 'elite' performance characteristics are.

During the development and evolution of these platforms not only were the authors somewhat educated in each other disciplines but found that the expectation of technology 
and its use developed within the traditional practice of coaches and athlete.

\section{ACKNOWLEDGMENTS}

This work is supported by Griffith University, the Australian Institute of Sport and the CRC for Microtechnology.

\section{REFERENCES}

[1] Hawley, J., Guidelines for laboratory and field testing of athletic potential and performance, Basic and Applied Sciences for Sports Medicine, edited by Maughan, R., Butterworth-Heinemann, Oxford, 1999

[2] H. Montoye, R. Washburn, S. Smais, A. Ertl, J. G. Webster, and F. J. Nagle,, Estimation of energy expenditure by a portable accelerometer, Med. Sci. Sports Exerc., vol. 15 , pp. $403407,1983$.
[3] Moe-Nilssen, R., and Helbostad, J. L., Estimation of gait cycle characteristics by trunk accelerometry. $\mathrm{J}$ Biomech, 37(1), 121-126, 2004

[4] James, D. A., Davey, N. and Gourdeas, L, An integrated platform for micro sensor data acquisition, storage and analysis, Proc. SPIE Vol. 5274, (SPIE, Bellingham, WA) 371-78, 2003

[5] Wixted, A. J.,James, D. A. and Thiel, D. V. Low Power Operating System and Wireless Networking for a Real Time Sensor Network", ICITA, Bathurst, Australia, Nov 2002 